POS $\quad$ PROCEEDINGS

\title{
New Results in Theories with Reduced Couplings
}

\author{
Sven Heinemeyer \\ Instituto de Física Teórica, Universidad Autónoma de Madrid, Cantoblanco, \\ 28049 Madrid, Spain \\ Campus of International Excellence UAM+CSIC, Cantoblanco, 28049 Madrid, Spain \\ Instituto de Física de Cantabria (CSIC-UC), E-39005 Santander, Spain \\ E-mail: Sven.Heinemeyer@cern.ch

\section{Myriam Mondragón} \\ Instituto de Física, Universidad Nacional Autónoma de México, A.P. 20-364, México 01000 \\ E-mail: myriamefisica.unam.mx
}

\section{Gregory Patellis*}

Physics Department, Nat. Technical University, 15780 Zografou, Athens, Greece

E-mail: patellisecentral.ntua.gr

\section{Nicholas Tracas}

Physics Department, Nat. Technical University, 15780 Zografou, Athens, Greece

E-mail: ntracecentral.ntua.gr

\section{George Zoupanos}

Physics Department, Nat. Technical University, 15780 Zografou, Athens, Greece Max-Planck Institut für Physik, Föhringer Ring 6, D-80805 München, Germany

E-mail: George.Zoupanos@cern.ch

The renormalization group invariant, to all orders in perturbation theory, relations among parameters consist the basis of the reduction of couplings concept. N=1 supersymmetric Grand Unified Theories can use the above concept, and even be finite at all loops. We review this idea as well as the resulting theories. We construct: (i) an $N=1 S U(5)$ minimal version, (ii) an all-loop finite $N=1 S U(5)$, (iii) a two-loop finite $N=1 S U(3)^{3}$ and finally (vi) a Minimal Supersymmetric Standard Model reduced version. The SUSY particle spectra predicted by these models are consistent with the LHC non-observation results.

Corfu Summer Institute 2019 'Workshops on Elementary Particle Physics and Gravity'

31 August - 25 September 2019

Corfu, Greece

\footnotetext{
*Speaker.
} 


\section{Introduction}

The reduction of couplings method [1-4] (see also [5-7]) seems a most promising method which relates independent, in a first view, parameters to a single, "primary" coupling. The method requires the original theory where is applied to be a renormalizable one, and the resulting relation among the parameters to be valid in all energy scales, i.e. are Renormalization Group Invariant (RGI).

The next (natural) step, after the introduction of a novel symmetry through a Grand Unified Theory (GUT) [8-13]), in order to achieve reduction of free parameters of the SM is the relation of the gauge to Yukawa sector (Gauge Yukawa Unification, GUY). This is the central characteristic of the reduction of couplings approach. According to that approach, being in a GUT environment, RGI relations are traced between the unification scale and the Planck one. One-loop uniqueness can guarantee all-loop validity of those relations. Moreover, RGI relations can be found which guarantee all order finiteness of a theory. The method has predicted the top quark mass in the finite $N=1 S U(5)$ model [15,16] as well as in the minimal $N=1 S U(5)$ one [14] before its experimental verification [17].

Since SuperSymmetry (SUSY) seems an essential ingredient for the reduction of couplings method, the extension to dimension-1 and -2 couplings, or in other words to the supersymmetry breaking sector (SSB), is unavoidable. The supergraph method and the spurion superfield technique played an important role for the progress in that sector, leading to all-loop finite models predicting the SUSY mass spectrum. The all-loop finite $N=1 S U(5)$ model [18] has given a prediction for the Higgs mass compatible with the experimental results [19-22] and a consistent (with the experimental non observation) heavy SUSY mass spectrum. Application of the method to the MMSM, gives consistent results for the Higgs, bottom, top masses and a heavy SUSY spectrum. The (new) FeynHiggs code [23-26] has been used for the calculation of the lightest Higgs. Special reference is made to the sum rule for the soft scalar masses (an RGI all-loop relation), which overcomes several phenomenological complications.

\section{Theoretical Basis}

Let us present the core idea of the reduction of couplings method. The target is to single out a basic parameter (the one we shall call the primary one), where all other parameters can be expressed in terms of this one through RGI relations. Such a relation has, in general, the form $\Phi\left(g_{1}, \cdots, g_{A}\right)=$ const. which should satisfy the following partial differential equation (PDE)

$$
\mu \frac{d \Phi}{d \mu}=\vec{\nabla} \Phi \cdot \vec{\beta}=\sum_{a=1}^{A} \beta_{a} \frac{\partial \Phi}{\partial g_{a}}=0
$$

where $\beta_{a}$ is the $\beta$-functions of $g_{a}$. The above PDE is equivalent to the following set of ordinary differential equations (ODEs), which are called Reduction Equations (REs) [2-4],

$$
\beta_{g} \frac{d g_{a}}{d g}=\beta_{a}, a=1, \cdots, A-1,
$$

where now $g$ is the primary coupling with its corresponding $\beta$-function. There are obviously $A-1$ relations in the form of $\Phi\left(g_{1}, \cdots, g_{A}\right)$ in order to express all other couplings in term of the primary one. 
The crucial demand is that the above REs admit power series solutions

$$
g_{a}=\sum_{n} \rho_{a}^{(n)} g^{2 n+1}
$$

which preserve perturbative renormalizability. Without this requirement, we just trade each "dependent" coupling with an integration constant. The power series, which are a set of special solutions, fix that constant. It is very important to point out that the uniqueness of such a solution can be already decided at the one-loop level [2-4]. In supersymmetric theories, where the asymptotic behaviour of several parameters are similar, the use of power series as solutions of the REs are justified. But, usually, the reduction is not "complete", which means that not all of the couplings can be reduced in favor of the primary one, leading to the so called "partial reduction" [27,28].

We proceed to the reduction scheme for massive parameters, which is far of being straightforward. A number of conditions is required (see for example [29]). Nevertheless, progress has been achieved, starting from [30], and finally we can introduce mass parameters and couplings carrying mass dimension $[31,32]$ in the same way as dimensionless couplings.

Concider the superpotential

$$
W=\frac{1}{2} \mu^{i j} \Phi_{i} \Phi_{j}+\frac{1}{6} C^{i j k} \Phi_{i} \Phi_{j} \Phi_{k},
$$

and the SSB sector Lagrangian

$$
-\mathscr{L}_{\text {SSB }}=\frac{1}{6} h^{i j k} \phi_{i} \phi_{j} \phi_{k}+\frac{1}{2} b^{i j} \phi_{i} \phi_{j}+\frac{1}{2}\left(m^{2}\right)_{i}^{j} \phi^{* i} \phi_{j}+\frac{1}{2} M \lambda_{i} \lambda_{i}+\text { h.c. },
$$

where $\phi_{i}$ 's are the scalar fields of the corresponding superfields $\Phi_{i}$ 's and $M$ is the gaugino mass which is described by $\lambda$.

Let us write down some well known relations:

(i) The $\beta$-function of the gauge coupling at one-loop level is given by [33-37]

$$
\beta_{g}^{(1)}=\frac{d g}{d t}=\frac{g^{3}}{16 \pi^{2}}\left[\sum_{i} T\left(R_{i}\right)-3 C_{2}(G)\right] .
$$

(ii) The anomalous dimension $\gamma_{j}^{(1)}{ }_{j}$, at a one-loop level, of a chiral superfield is

$$
\gamma_{j}^{(1) i}=\frac{1}{32 \pi^{2}}\left[C^{i k l} C_{j k l}-2 g^{2} C_{2}\left(R_{i}\right) \delta_{j}^{i}\right] .
$$

(iii) The $\beta$-functions of $C_{i j k}$ 's, at one-loop level, following the $N=1$ non-renormalization theorem [38-40], are expressed in terms of the anomalous dimensions of the fields involved

$$
\beta_{C}^{i j k}=\frac{d C_{i j k}}{d t}=C_{i j l} \gamma_{k}^{l}+C_{i k l} \gamma_{j}^{l}+C_{j k l} \gamma_{i}^{l}
$$

We proceed by assuming that the REs admit power series solutions:

$$
C^{i j k}=g \sum_{n=0} \rho_{(n)}^{i j k} g^{2 n} .
$$


Trying to obtain all-loop results we turn to relations among $\beta$-functions. The spurion technique [40-44] gives all-loop relations among SSB $\beta$-functions [45-51]. Then, assuming that the reduction of $C^{i j k}$ is possible to all orders

$$
\frac{d C^{i j k}}{d g}=\frac{\beta_{C}^{i j k}}{\beta_{g}},
$$

as well as for $h^{i j k}$

$$
h^{i j k}=-M \frac{d C(g)^{i j k}}{d \ln g},
$$

then it can be proven $[52,53]$ that the following relations are all-loop RGI

$$
\begin{aligned}
M & =M_{0} \frac{\beta_{g}}{g}, \\
h^{i j k} & =-M_{0} \beta_{C}^{i j k}, \\
b^{i j} & =-M_{0} \beta_{\mu}^{i j}, \\
\left(m^{2}\right)_{j}^{i} & =\frac{1}{2}\left|M_{0}\right|^{2} \mu \frac{d \gamma_{j}^{i}}{d \mu},
\end{aligned}
$$

where $M_{0}$ is an arbitrary reference mass scale to be specified (note that in both assumptions we do not rely on specific solutions of these equations).

As a next step we substitute the last equation, Eq.(2.15), by a more general RGI sum rule that holds to all orders [54]

$$
\begin{aligned}
m_{i}^{2}+m_{j}^{2}+m_{k}^{2}=|M|^{2}\{ & \left.\frac{1}{1-g^{2} C_{2}(G) /\left(8 \pi^{2}\right)} \frac{d \ln C^{i j k}}{d \ln g}+\frac{1}{2} \frac{d^{2} \ln C^{i j k}}{d(\ln g)^{2}}\right\} \\
& +\sum_{l} \frac{m_{l}^{2} T\left(R_{l}\right)}{C_{2}(G)-8 \pi^{2} / g^{2}} \frac{d \ln C^{i j k}}{d \ln g},
\end{aligned}
$$

which leads to the following one-loop relation

$$
m_{i}^{2}+m_{j}^{2}+m_{k}^{2}=|M|^{2} .
$$

Finally, note that in the case of product gauge groups, Eq.(2.12) takes the form

$$
M_{i}=\frac{\beta_{g_{i}}}{g_{i}} M_{0}
$$

where $i$ denotes the group of the product. This will be used in the Reduced MSSM case.

\section{Finiteness in $N=1$ Supersymmetric Gauge Theories}

Consider an $N=1$ globally supersymmetric gauge theory, which is chiral and anomaly free, where $G$ is the gauge group and $g$ the associated gauge coupling. The theory has the superpotential of Eq.(2.4), while the one-loop gauge and $C_{i j k} \mathrm{~s} \beta$-function are given by Eq.(2.6) and Eq.(2.8) respectively and the one-loop anomalous dimensions of the chiral superfields by Eq.(2.7).

Demanding the vanishing of all one-loop $\beta$-functions, Eqs. $(2.6,2.7)$ lead to the relations

$$
\begin{aligned}
& \sum_{i} T\left(R_{i}\right)=3 C_{2}(G), \\
& C^{i k l} C_{j k l}=2 \delta_{j}^{i} g^{2} C_{2}\left(R_{i}\right) .
\end{aligned}
$$


The finiteness conditions for an $N=1$ supersymmetric theory with $S U(N)$ associated group is found in [55] while discussion of the no-charge renormalization and anomaly free requiremnts can be found in [56]. It should be noted that conditions (3.1) and (3.2) are necessary and sufficient to ensure finiteness at the two-loop level [33-37].

The requirement of finiteness, at the one-loop level, in softly broken SUSY theories demands additional constraints among the soft terms of the SSB sector [57], while, once more, these oneloop requirements assure two-loop finiteness, too [58]. These conditions impose restrictions on the irreducible representations $R_{i}$ of the gauge group $G$ as well as on the Yukawa couplings. For example, since $U(1)$ s are not compatible with condition (3.1), the MSSM is excluded. Therefore, a GUT is initially required with the MSSM being its low energy theory. Also, since condition (3.2) forbids the appearance of gauge singlets $\left(C_{2}(1)=0\right)$, F-type spontaneous symmetry breaking [59] are not compatible with finiteness. Finally, D-type spontaneous breaking [60] is also incompatible since it requires a $U(1)$ group.

The non trivial point is that the relations among couplings (gauge and Yukawa) which are imposed by the conditions (3.1) and (3.2) should hold at any energy scale. The necessary and sufficient condition is to require that such relations are solutions to the REs (see Eq. (2.10))

$$
\beta_{g} \frac{d C_{i j k}}{d g}=\beta_{i j k}
$$

holding at all orders. We note, once more, that the existence of one-loop level power series solution guarantees the all-order series.

There exist the following theorem [61,62] which points down which are the the necessary and sufficient conditions in order for an $N=1$ SUSY theory to be all-loop finite. In refs [61-67] it was shown that for an $N=1$ SUSY Yang-Mills theory, based on a simple gauge group, if the following four conditions are fulfilled:

(i) No gauge anomaly is present.

(ii) The $\beta$-function of the gauge coupling is zero at one-loop level

$$
\beta_{g}^{(1)}=0=\sum_{i} T\left(R_{i}\right)-3 C_{2}(G)
$$

(iii) The condition of vanishing for the one-loop anomalous dimensions of matter fields,

$$
\gamma_{j}^{(1) i}=0=\frac{1}{32 \pi^{2}}\left[C^{i k l} C_{j k l}-2 g^{2} C_{2}(R) \delta_{j}^{i}\right]
$$

admits solution of the form

$$
C_{i j k}=\rho_{i j k} g, \quad \rho_{i j k} \in \mathbb{C} .
$$

(iv) When considered as solutions of vanishing Yukawa $\beta$-functions (at one-loop order), i.e. $\beta_{i j k}=$ 0 , the above solutions are isolated and non-degenerate.

Then, each of the solutions in Eq.(3.6) can be extended uniquely to a formal power series in $g$, and the associated super Yang-Mills models depend on the single coupling constant $g$ with a vanishing, at all orders, $\beta$-function.

While the validity of the above cannot be extended to non-SUSY theories, it should be noted that reduction of couplings and finiteness are intimately related. 


\section{The Reduction of Coupling Method in Phenomenological Models}

In this section we apply the method to four interesting phenomenological models, namely (i) the Minimal $N=1$ Supersymmetric $S U(5)$, (ii) the Finite $N=1$ Supersymmetric $S U(5)$, (iii) the Finite $S U(N)^{3}$ and (iv) the MSSM. Discussion on the predictions for quark masses, the light Higgs boson mass, the SUSY breaking scale (defined as the geometric mean of stops), and the full supersymmetric spectra are discussed in Sect. 6.

\subsection{The Minimal $N=1$ Supersymmetric $S U(5)$ Model}

We start with the partial reduction of the $N=1$ SUSY SU(5) model [14,30]. Our notation is as follows: $\Psi^{I}(\mathbf{1 0})$ and $\Phi^{I}(\overline{\mathbf{5}})$ refer to the three generations of lepton and quarks $(I=1,2,3)$, the adjoint $\Sigma(\mathbf{2 4})$ breaks $S U(5)$ to $S U(3)_{\mathrm{C}} \times S U(2)_{\mathrm{L}} \times U(1)_{\mathrm{Y}}$ and $\bar{H}(\overline{\mathbf{5}})$ represent the two Higgs superfields for the electroweak symmetry breaking (ESB) $[68,69]$. The choice of using only one set of $(\mathbf{5}+\overline{\mathbf{5}})$ for the ESB renders the model asymptoticaly free (i.e. $\left.\beta_{g}<0\right)$. The superpotential of the model is described by

$$
\begin{aligned}
W & =\frac{g_{t}}{4} \varepsilon^{\alpha \beta \gamma \delta \tau} \Psi_{\alpha \beta}^{(3)} \Psi_{\gamma \delta}^{(3)} H_{\tau}+\sqrt{2} g_{b} \Phi^{(3) \alpha} \Psi_{\alpha \beta}^{(3)} \bar{H}^{\beta}+\frac{g_{\lambda}}{3} \Sigma_{\alpha}^{\beta} \Sigma_{\beta}^{\gamma} \Sigma_{\gamma}^{\alpha}+g_{f} \bar{H}^{\alpha} \Sigma_{\alpha}^{\beta} H_{\beta} \\
& +\frac{\mu_{\Sigma}}{2} \Sigma_{\alpha}^{\gamma} \Sigma_{\gamma}^{\alpha}+\mu_{H} \bar{H}^{\alpha} H_{\alpha} .
\end{aligned}
$$

where only the third generation Yukawa couplings are taken into account. The indices $\alpha, \beta, \gamma, \delta, \tau$ are $S U(5)$ ones. A detailed presentation of the model can be found in [70] and in [71,72].

Our primary coupling is the gauge one $g$. In this model the gauge-Yukawa unification can be achieved through two sets of solutions which are asympotically free [70]:

$$
\begin{aligned}
& a: g_{t}=\sqrt{\frac{2533}{2605}} g+\mathscr{O}\left(g^{3}\right), g_{b}=\sqrt{\frac{1491}{2605}} g+\mathscr{O}\left(g^{3}\right), g_{\lambda}=0, g_{f}=\sqrt{\frac{560}{521}} g+\mathscr{O}\left(g^{3}\right), \\
& b: g_{t}=\sqrt{\frac{89}{65}} g+\mathscr{O}\left(g^{3}\right), g_{b}=\sqrt{\frac{63}{65}} g+\mathscr{O}\left(g^{3}\right), g_{\lambda}=0, g_{f}=0 .
\end{aligned}
$$

where the higher order terms denote uniquely computable power series in $g$. Let us note that the reduction of the dimensionless sector is independent of the dimensionful one. These solutions describe the boundaries of a RGI surface in the parameter space which is AF and where $g_{f}$ and $g_{\lambda}$ could be different from zero. Therefore, a partial reduction is possible where $g_{\lambda}$ and $g_{f}$ are independent (non-vanishing) parameters without endangering AF. The proton decay constraints favour solution $a$, therefore we choose this one for our discussion. ${ }^{1}$

The SSB Lagrangian is

$$
\begin{aligned}
-\mathscr{L}_{\text {soft }} & =m_{H_{u}}^{2} \hat{H}^{* \alpha} \hat{H}_{\alpha}+m_{H_{d}}^{2} \hat{\bar{H}}_{\alpha}^{*} \hat{\bar{H}}^{\alpha}+m_{\Sigma}^{2} \hat{\Sigma}_{\beta}^{\dagger} \alpha \hat{\Sigma}_{\alpha}^{\beta}+\sum_{I=1,2,3}\left[m_{\Phi^{I}}^{2} \hat{\Phi}_{\alpha}^{*(I)} \hat{\Phi}^{(I) \alpha}\right. \\
& \left.+m_{\Psi^{I}}^{2} \hat{\Psi}^{\dagger}(I) \alpha \beta \hat{\Psi}_{\beta \alpha}^{(I)}\right]+\left\{\frac{1}{2} M \lambda \lambda+B_{H} \hat{\bar{H}}^{\alpha} \hat{H}_{\alpha}+B_{\Sigma} \hat{\Sigma}_{\beta}^{\alpha} \hat{\Sigma}_{\alpha}^{\beta}+h_{f} \hat{\bar{H}}^{\alpha} \hat{\Sigma}_{\alpha}^{\beta} \hat{H}_{\beta}\right. \\
& \left.+\frac{h_{\lambda}}{3} \hat{\Sigma}_{\alpha}^{\beta} \hat{\Sigma}_{\beta}^{\gamma} \hat{\Sigma}_{\gamma}^{\alpha}+\frac{h_{t}}{4} \varepsilon^{\alpha \beta \gamma \delta \tau} \hat{\Psi}_{\alpha \beta}^{(3)} \hat{\Psi}_{\gamma \delta}^{(3)} \hat{H}_{\tau}+\sqrt{2} h_{b} \hat{\Phi}^{(3) \alpha} \hat{\Psi}_{\alpha \beta}^{(3)} \hat{H}^{\beta}+\text { h.c. }\right\}
\end{aligned}
$$

\footnotetext{
${ }^{1} g_{\lambda}=0$ is inconsistent, but $g_{\lambda}<\sim 0.005$ is necessary in order for the proton decay constraint [73] to be satisfied. A small $g_{\lambda}$ is expected not to affect the prediction of unification of SSB parameters.
} 
where the hat denotes the scalar components of the chiral superfields. The parameters $M, \mu_{\Sigma}$ and $\mu_{H}$ are treated as independent ones, since they cannot be reduced in a suitable form. The lowest-order reduction for the parameters of the SSB Lagrangian are given by:

$$
\begin{gathered}
B_{H}=\frac{1029}{521} \mu_{H} M, B_{\Sigma}=-\frac{3100}{521} \mu_{\Sigma} M, \\
h_{t}=-g_{t} M, h_{b}=-g_{b} M, h_{f}=-g_{f} M, h_{\lambda}=0, \\
m_{H_{u}}^{2}=-\frac{569}{521} M^{2}, m_{H_{d}}^{2}=-\frac{460}{521} M^{2}, m_{\Sigma}^{2}=\frac{1550}{521} M^{2}, \\
m_{\Phi^{3}}^{2}=\frac{436}{521} M^{2}, m_{\Phi^{1,2}}^{2}=\frac{8}{5} M^{2}, m_{\Psi^{3}}^{2}=\frac{545}{521} M^{2}, m_{\Psi^{1,2}}^{2}=\frac{12}{5} M^{2} .
\end{gathered}
$$

We choose the gaugino mass $M$ for characterizing the SUSY breaking scale. Finally, we note that (i) $B_{\Sigma}$ and $B_{H}$ are treated as independent parameters without spoiling the one-loop reduction solution of Eq.(4.5) and (ii) the sum rule still holds despite the specific relations among the gaugino mass and the soft scalar masses.

\subsection{The Finite $N=1$ Supersymmetric $S U(5)$ Model}

We proceed now to the finite to all-orders $S U(5)$ gauge theory, where the reduction of couplings is restricted to the third generation. This specific Finite Unified Theory (FUT) was in agreement with the experimental constraints at the time [18] and has predicted, almost five years before its discovery, the light Higgs mass in the range of $121-126 \mathrm{GeV} .{ }^{2}$ The particle content of the model has three $(\overline{\mathbf{5}}+\mathbf{1 0})$ supermultiplets for the three generations of leptons and quarks, while the Higgs sector consists of four supermultiplets $(\overline{\mathbf{5}}+\mathbf{5})$ and one $\mathbf{2 4}$. The finite $S U(5)$ group is broken to MSSM, which of course in no longer a finite theory [14-16,74-76].

In order for this finite to all-orders $S U(5)$ model to achieve Gauge Yukawa Unification (GYU) should have the following characteristics:

(i) The one-loop anomalous dimensions are diagonal i.e., $\gamma_{i}^{(1) j} \propto \delta_{i}^{j}$.

(ii) The fermions of the $\overline{\mathbf{5}}_{i}$ and $\mathbf{1 0}_{i}(i=1,2,3)$ are not coupled to the $\mathbf{2 4}$.

(iii) The pair of the MSSM Higgs doublets are mostly consisted from the 5 and $\overline{5}$ Higgs and couple to the third generation

The superpotential of the model, with an enhanced symmetry due to the reduction of couplings, is given by $[77,78]$ :

$$
\begin{aligned}
W & =\sum_{i=1}^{3}\left[\frac{1}{2} g_{i}^{u} \mathbf{1 0}_{i} \mathbf{1 0}_{i} H_{i}+g_{i}^{d} \mathbf{1 0}_{i} \overline{\mathbf{5}}_{i} \bar{H}_{i}\right]+g_{23}^{u} \mathbf{1 0}_{2} \mathbf{1 0}_{3} H_{4} \\
& +g_{23}^{d} \mathbf{1 0}_{2} \overline{\mathbf{5}}_{3} \bar{H}_{4}+g_{32}^{d} \mathbf{1 0}_{3} \overline{\mathbf{5}}_{2} \bar{H}_{4}+g_{2}^{f} H_{2} \mathbf{2 4} \bar{H}_{2}+g_{3}^{f} H_{3} \mathbf{2 4} \bar{H}_{3}+\frac{g^{\lambda}}{3}(\mathbf{2 4})^{3} .
\end{aligned}
$$

\footnotetext{
${ }^{2}$ Improved Higgs mass calculations would yield a different interval, still compatible with current experimental data (see below).
} 
Discussion of the model with a more detailed description can be found in [14-16]. The nondegenerate and isolated solutions to the vanishing of $\gamma_{i}^{(1)}$ are:

$$
\begin{aligned}
& \left(g_{1}^{u}\right)^{2}=\frac{8}{5} g^{2},\left(g_{1}^{d}\right)^{2}=\frac{6}{5} g^{2},\left(g_{2}^{u}\right)^{2}=\left(g_{3}^{u}\right)^{2}=\frac{4}{5} g^{2}, \\
& \left(g_{2}^{d}\right)^{2}=\left(g_{3}^{d}\right)^{2}=\frac{3}{5} g^{2},\left(g_{23}^{u}\right)^{2}=\frac{4}{5} g^{2},\left(g_{23}^{d}\right)^{2}=\left(g_{32}^{d}\right)^{2}=\frac{3}{5} g^{2}, \\
& \left(g^{\lambda}\right)^{2}=\frac{15}{7} g^{2},\left(g_{2}^{f}\right)^{2}=\left(g_{3}^{f}\right)^{2}=\frac{1}{2} g^{2},\left(g_{1}^{f}\right)^{2}=0,\left(g_{4}^{f}\right)^{2}=0 .
\end{aligned}
$$

We have also the relation $h=-M C$, while the sum rules lead to:

$$
m_{H_{u}}^{2}+2 m_{\mathbf{1 0}}^{2}=M^{2}, m_{H_{d}}^{2}-2 m_{\mathbf{1 0}}^{2}=-\frac{M^{2}}{3}, m_{\overline{\mathbf{5}}}^{2}+3 m_{\mathbf{1 0}}^{2}=\frac{4 M^{2}}{3} .
$$

Therefore, we only have two free parameters, namely $m_{10}$ and $M$ in the dimensionful sector.

When $S U(5)$ breaks down to the MSSM, a suitable rotation in the Higgs sector [15, 16, 7982], permits only a pair of Higgs doublets (coupled mostly to the third family) to remain light and acquire vev's. Avoiding fast proton decay is achieved with the usual doublet-triplet splitting, although different from the one applied to the minimal $S U(5)$ due to the extended Higgs sector of the finite model. Therefore, below the GUT scale we get the MSSM where the third generation is given by the finiteness conditions while the first two remain unrestricted.

\subsection{The Finite $S U(N)^{3}$ Model}

We proceed now to construct a FUT based on a product gauge group. Concider an $N=1$ SUSY theory with $S U(N)_{1} \times S U(N)_{2} \times \cdots \times S U(N)_{k}$ having $n_{f}$ families transforming as $\left(N, N^{*}, 1, \ldots, 1\right)+$ $\left(1, N, N^{*}, \ldots, 1\right)+\cdots+\left(N^{*}, 1,1, \ldots, N\right)$. Then, the first order coefficient of the $\beta$-function, for each $S U(N)$ group is:

$$
b=\left(-\frac{11}{3}+\frac{2}{3}\right) N+n_{f}\left(\frac{2}{3}+\frac{1}{3}\right)\left(\frac{1}{2}\right) 2 N=-3 N+n_{f} N .
$$

Demanding finiteness, i.e. $b=0$, we are led to the choice $n_{f}=3$. Phenomenological reasons lead to the choice of the $S U(3)_{C} \times S U(3)_{L} \times S U(3)_{R}$ model, discussed in Ref. [83], while a detailed discussion of the general well known example can be found in Ref. [84-87]. The leptons and quarks transform as:

$$
q=\left(\begin{array}{lll}
d & u & h \\
d & u & h \\
d & u & h
\end{array}\right) \sim\left(3,3^{*}, 1\right), \quad q^{c}=\left(\begin{array}{lll}
d^{c} & d^{c} & d^{c} \\
u^{c} & u^{c} & u^{c} \\
h^{c} & h^{c} & h^{c}
\end{array}\right) \sim\left(3^{*}, 1,3\right), \quad \lambda=\left(\begin{array}{ccc}
N & E^{c} & v \\
E & N^{c} & e \\
v^{c} & e^{c} & S
\end{array}\right) \sim\left(1,3,3^{*}\right)
$$

where $h$ are down-type quarks acquiring masses close to $M_{G U T}$. A cyclic $Z_{3}$ symmetry is imposed on the multiplets to achieve equal gauge couplings at the GUT scale and in that case the vanishing of the first-order $\beta$-function is satisfied. Continuing to the vanishing of the anomalous dimension of all the fields (see Eq.(3.2)), we note that there are two trilinear invariant terms in the superpotential, namely:

$$
f \operatorname{Tr}\left(\lambda q^{c} q\right)+\frac{1}{6} f^{\prime} \varepsilon_{i j k} \varepsilon_{a b c}\left(\lambda_{i a} \lambda_{j b} \lambda_{k c}+q_{i a}^{c} q_{j b}^{c} q_{k c}^{c}+q_{i a} q_{j b} q_{k c}\right)
$$


with $f$ and $f^{\prime}$ the corresponding Yukawa couplings. The superfields $\left(\tilde{N}, \tilde{N}^{c}\right)$ obtain vev's and provide masses to leptons and quarks

$$
m_{d}=f\langle\tilde{N}\rangle, m_{u}=f\left\langle\tilde{N}^{c}\right\rangle, m_{e}=f^{\prime}\langle\tilde{N}\rangle, m_{v}=f^{\prime}\left\langle\tilde{N}^{c}\right\rangle .
$$

Having three families, $11 f$ couplings and $10 f^{\prime}$ couplings are present in the most general superpotential. Demanding the vanishing of all superfield anomalous dimensions, 9 conditions are imposed

$$
\sum_{j, k} f_{i j k}\left(f_{l j k}\right)^{*}+\frac{2}{3} \sum_{j, k} f_{i j k}^{\prime}\left(f_{l j k}^{\prime}\right)^{*}=\frac{16}{9} g^{2} \delta_{i l}
$$

where

$$
f_{i j k}=f_{j k i}=f_{k i j}, \quad f_{i j k}^{\prime}=f_{j k i}^{\prime}=f_{k i j}^{\prime}=f_{i k j}^{\prime}=f_{k j i}^{\prime}=f_{j i k}^{\prime} .
$$

The masses of leptons and quarks are acquired from the vev's of the scalar parts of the superfields $\tilde{N}_{1,2,3}$ and $\tilde{N}_{1,2,3}^{c}$.

At $M_{\mathrm{GUT}}$ the $S U(3)^{3}$ FUT breaks ${ }^{3}$ to MSSM, where as was already mentioned, both Higgs doublets couple mostly to the third generation. The FUT breaking leaves its mark in the form of Eq.(4.13), i.e. boundary conditions on the gauge and Yukawa couplings, the relation $h=-M f$ and finally the soft scalar mass sum rule at $M_{\mathrm{GUT}}$. In this specific model this rule takes the form:

$$
m_{H_{u}}^{2}+m_{\tilde{t}^{c}}^{2}+m_{\tilde{q}}^{2}=M^{2}=m_{H_{d}}^{2}+m_{\tilde{b}^{c}}^{2}+m_{\tilde{q}}^{2} .
$$

The model is finite to all-orders if the solution of Eq.(4.13) is both isolated and unique. Then, $f^{\prime}=0$ and we have the relations

$$
f^{2}=f_{111}^{2}=f_{222}^{2}=f_{333}^{2}=\frac{16}{9} g^{2} .
$$

Since all $f^{\prime}$ vanish, in one-loop order, the lepton masses vanish. Since these masses, even radiatively, cannot be produced because of the finiteness conditions, we are faced with a problem which needs further study. If the solution of Eq.(4.13) is unique but not isolated (i.e. parametric), we can have non zero $f^{\prime}$ leading to non-vanishing lepton masses and at the same time achieving two-loop finiteness. In that case the set of conditions restricting the Yukawa couplings read:

$$
f^{2}=r\left(\frac{16}{9}\right) g^{2}, \quad f^{\prime 2}=(1-r)\left(\frac{8}{3}\right) g^{2},
$$

where $r$ parametrises the different solutions and as such is a free parameter. It should be noted that we use the sum rule as boundary condition for the soft scalar masses.

\subsection{The Reduced MSSM}

We end up with the application of the method of coupling reduction to a version of the MSSM, where a covering GUT is assumed. The original partial reduction can be found in ref. [90,91] where only the third fermionic generation is considered. Following this restriction, the superpotential reads:

$$
W=Y_{t} H_{2} Q t^{c}+Y_{b} H_{1} Q b^{c}+Y_{\tau} H_{1} L \tau^{c}+\mu H_{1} H_{2},
$$

\footnotetext{
${ }^{3}[88,89]$ and refs therein discuss in detail the spontaneous breaking of $S U(3)^{3}$.
} 
where $Y_{t, b, \tau}$ and $h_{t, b, \tau}$ refer only to the third family, and the SSB Lagrangian is given by

$$
\begin{aligned}
-\mathscr{L}_{\text {SSB }} & =\sum_{\phi} m_{\phi}^{2} \hat{\phi^{*}} \hat{\phi}+\left[m_{3}^{2} \hat{H}_{1} \hat{H}_{2}+\sum_{i=1}^{3} \frac{1}{2} M_{i} \lambda_{i} \lambda_{i}+\text { h.c }\right] \\
& +\left[h_{t} \hat{H_{2}} \hat{Q} \hat{t}^{c}+h_{b} \hat{H_{1}} \hat{Q} \hat{b}^{c}+h_{\tau} \hat{H}_{1} \hat{L} \hat{\tau}^{c}+\text { h.c. }\right],
\end{aligned}
$$

We start with the dimensionless sector and consider initially the top and bottom Yuakwa couplings and the strong gauge one. The rest of the couplings will be treated as corrections. Their running behaviour, as corrections to the strong coupling, is understood [27]. If $Y_{(t, b)}^{2} /(4 \pi) \equiv \alpha_{(t, b)}$, the REs and the Yukawa RGEs give

$$
\alpha_{i}=G_{i}^{2} \alpha_{3}, \quad \text { where } G_{i}^{2}=\frac{1}{3}, \quad i=t, b .
$$

If the tau Yukawa is included in the reduction, the corresponding $G^{2}$ coefficient for tau turns negative [92], explaining why this coupling is treated also as a correction.

We assume that the ratios of the top and bottom Yukawa to the strong coupling are constant on the GUT scale, i.e. they have negligible scale dependence,

$$
\frac{d}{d g_{3}}\left(\frac{Y_{t, b}^{2}}{g_{3}^{2}}\right)=0,
$$

Then, including the corrections from the $S U(2), U(1)$ and tau couplings, at the GUT scale, the coefficients $G_{t, b}^{2}$ become:

$$
G_{t}^{2}=\frac{1}{3}+\frac{71}{525} \rho_{1}+\frac{3}{7} \rho_{2}+\frac{1}{35} \rho_{\tau}, \quad G_{b}^{2}=\frac{1}{3}+\frac{29}{525} \rho_{1}+\frac{3}{7} \rho_{2}-\frac{6}{35} \rho_{\tau}
$$

where

$$
\rho_{1,2}=\frac{g_{1,2}^{2}}{g_{3}^{2}}=\frac{\alpha_{1,2}}{\alpha_{3}}, \quad \rho_{\tau}=\frac{g_{\tau}^{2}}{g_{3}^{2}}=\frac{\frac{Y_{\tau}^{2}}{4 \pi}}{\alpha_{3}}
$$

We shall treat Eqs.(4.20) as boundary conditions at the GUT scale.

Going to the two-loop level, we assume that the corrections take the following form:

$$
\alpha_{i}=G_{i}^{2} \alpha_{3}+J_{i}^{2} \alpha_{3}^{2}, \quad i=t, b .
$$

Then, the two-loop coefficients, $J_{i}$, including the corrections from the gauge and the tau Yukawa couplings, are:

$$
J_{t}^{2}=\frac{1}{4 \pi} \frac{N_{t}}{D}, \quad J_{b}^{2}=\frac{1}{4 \pi} \frac{N_{b}}{5 D}
$$

where $D, N_{t}$ and $N_{b}$ are known quantities which can be found in ref. [93].

Proceeding to the the SSB Lagrangian, Eq.(4.19), and the dimension-one parameters, i.e the trilinear couplings $h_{t, b, \tau}$, we first reduce $h_{t, b}$ and we get

$$
h_{i}=c_{i} Y_{i} M_{3}=c_{i} G_{i} M_{3} g_{3}, \text { where } c_{i}=-1 \quad i=t, b,
$$


and $M_{3}$ is the gluino mass. Adding the corrections from the gauge and the tau couplings we have

$$
c_{t}=-\frac{A_{A} A_{b b}+A_{t b} B_{B}}{A_{b t} A_{t b}-A_{b b} A_{t t}}, \quad c_{b}=-\frac{A_{A} A_{b t}+A_{t t} B_{B}}{A_{b t} A_{t b}-A_{b b} A_{t t}} .
$$

Again, $A_{t t}, A_{b b}$ and $A_{t b}$ can be found in ref. [93].

We end up with the soft scalar masses $m_{\phi}^{2}$ of the SSB Lagrangian. Assuming the relations $m_{i}^{2}=c_{i} M_{3}^{2}\left(i=Q, u, d, H_{u}, H_{d}\right)$, and adding the corrections from the gauge, the tau couplings and $h_{\tau}$, we get

$$
c_{Q}=-\frac{c_{Q \mathrm{Num}}}{D_{m}}, \quad c_{u}=-\frac{1}{3} \frac{c_{u \mathrm{Num}}}{D_{m}}, \quad c_{d}=-\frac{c_{d \mathrm{Num}}}{D_{m}}, \quad c_{H_{u}}=-\frac{2}{3} \frac{c_{H u \mathrm{Num}}}{D_{m}}, \quad c_{H_{d}}=-\frac{c_{H d \mathrm{Num}}}{D_{m}},
$$

where $D_{m}, c_{Q \mathrm{Num}}, c_{u \mathrm{Num}}, c_{d \mathrm{Num}}, c_{H u \mathrm{Num}}, c_{H d \mathrm{Num}}$ and the complete analysis are again given in ref. [93].

If only the reduced system were used, i.e. the strong, top and bottom Yukawa couplings as well as the $h_{t}$ and $h_{b}$, the coefficients turn to be

$$
c_{Q}=c_{u}=c_{d}=\frac{2}{3}, \quad c_{H_{u}}=c_{H_{d}}=-1 / 3,
$$

which clearly obey the sum rules

$$
\frac{m_{Q}^{2}+m_{u}^{2}+m_{H_{u}}^{2}}{M_{3}^{2}}=c_{Q}+c_{u}+c_{H_{u}}=1, \quad \frac{m_{Q}^{2}+m_{d}^{2}+m_{H_{d}}^{2}}{M_{3}^{2}}=c_{Q}+c_{d}+c_{H_{d}}=1 .
$$

We finish this section with an essential point for the gaugino masses. The application of the Hisano-Shiftman relation, Eq.(2.12), is made for each gaugino mass as a boundary condition with unified gauge coupling at $M_{\mathrm{GUT}}$ scale. Then, at one-loop level, the gaugino mass depends on the one-loop coefficient of the corresponding $\beta$-function and an arbitrary $M_{0}, \quad M_{i}=b_{i} M_{0}$. This fact permits, with a suitable choice of $M_{0}$, to have the gluino mass equal to the unified gaugino mass, while the gauginos masses of the other two gauge groups are given by the gluino mass multiplied by the ratio of the appropriate one-loop $\beta$ coefficient.

\section{Phenomenological Constraints}

In this section we shall briefly review several experimental constraints that were applied to our phenomenological analysis. The used values do not correspond to the latest experimental results. However, this has a negligible impact on our analysis.

In our models we evaluate the pole mass of the top quark while the bottom quark mass is evaluated at the $M_{Z}$ scale (to avoid uncertainties to its pole mass). The experimental values, taken from ref. [94] are:

$$
m_{t}^{\exp }=(173.1 \pm 0.9) \mathrm{GeV} \quad, \quad m_{b}\left(M_{Z}\right)=2.83 \pm 0.10 \mathrm{GeV} .
$$

We interpret the Higgs-like particle discovered in July 2012 by ATLAS and CMS [19,95] as the light $\mathscr{C} \mathscr{P}$-even Higgs boson of the MSSM [96-98]. The (SM) Higgs boson experimental average mass is [94] ${ }^{4}$

$$
M_{H}^{\exp }=125.10 \pm 0.14 \mathrm{GeV} .
$$

\footnotetext{
${ }^{4}$ This is the latest available LHC combination. More recent measurements confirm this value.
} 
The theoretical uncertainty $[23,24]$, however, for the prediction of $M_{h}$ in the MSSM dominates the total uncertainty, since it is much larger than the experimental one. In our follwing analyses we shall use the new FeynHiggs code [23-25] (Version 2.16.0) to predict the Higgs mass. FeynHiggs evaluates the Higgs masses based on a combination of fixed order diagrammatic calculations and resummation of the (sub)leading logarithmic contributions at all orders. This provides a reliable $M_{h}$ even for a large SUSY scale. This new version gives a downward shift on the Higgs mass $M_{h}$ of the order of $\mathscr{O}(2 \mathrm{GeV})$ for large SUSY masses, while computes the Higgs mass uncertainty point by point. The theoretical uncertainty calculated is added linearly to the experimental error in Eq.(5.2).

We also consider the following four flavour observables where SUSY has non-negligible impact. For the branching ratio $\operatorname{BR}(b \rightarrow s \gamma)$ we take a value from $[99,100]$, while for the branching ratio $\mathrm{BR}\left(B_{s} \rightarrow \mu^{+} \mu^{-}\right)$we use a combination of [101-105]:

$$
\frac{\mathrm{BR}(b \rightarrow s \gamma)^{\exp }}{\mathrm{BR}(b \rightarrow s \gamma)^{\mathrm{SM}}}=1.089 \pm 0.27 \quad, \quad \mathrm{BR}\left(B_{s} \rightarrow \mu^{+} \mu^{-}\right)=(2.9 \pm 1.4) \times 10^{-9}
$$

For the $B_{u}$ decay to $\tau \nu$ we use $[100,106,107]$ and for $\Delta M_{B_{s}}$ we use $[108,109]$ :

$$
\frac{\mathrm{BR}\left(B_{u} \rightarrow \tau v\right)^{\exp }}{\mathrm{BR}\left(B_{u} \rightarrow \tau v\right)^{\mathrm{SM}}}=1.39 \pm 0.69 \quad, \quad \frac{\Delta M_{B_{s}}^{\exp }}{\Delta M_{B_{s}}^{\mathrm{SM}}}=0.97 \pm 0.2
$$

\section{Numerical Analysis}

In this section we review the results of the phenomenological analysis of the models reviewed above. The full analysis can be found in our recent work [110], where it was shown - among others - that for the Minimal $S U(5)$ model the bottom mass is in agreement with the experimental results only at the $4 \sigma$ level. Furthermore, no model fulfills the current Cold Dark Matter (CDM) bounds, since the relic abundance is either too high (in all three GUTs) or too low (Reduced MSSM). Alternative ways are proposed for the two $S U(5)$ models in [110], while a similar R-parity violation should be considered for the other two as well.

\subsection{Finite $N=1 S U(5)$}

As discussed in Subsection 4.2, we present the full particle spectrum predicted in the Finite $N=1$ supersymmetric $S U(5)$ model. Conditions set by finiteness do not restrict the renormalization properties at low energies, so we are left with boundary conditions on the gauge and Yukawa couplings (4.7), the $h=-M C$ relation and the soft scalar-mass sum rule at $M_{\mathrm{GUT}}$. In Fig. $1, m_{b}\left(M_{Z}\right)$ and $m_{t}$ are shown as functions of the unified gaugino mass $M$. The orange (blue) lines denote the $2 \sigma(3 \sigma)$ experimental uncertainties. The only phenomenologically viable option is to consider $\mu<0$ (as shown in [110-117]. 

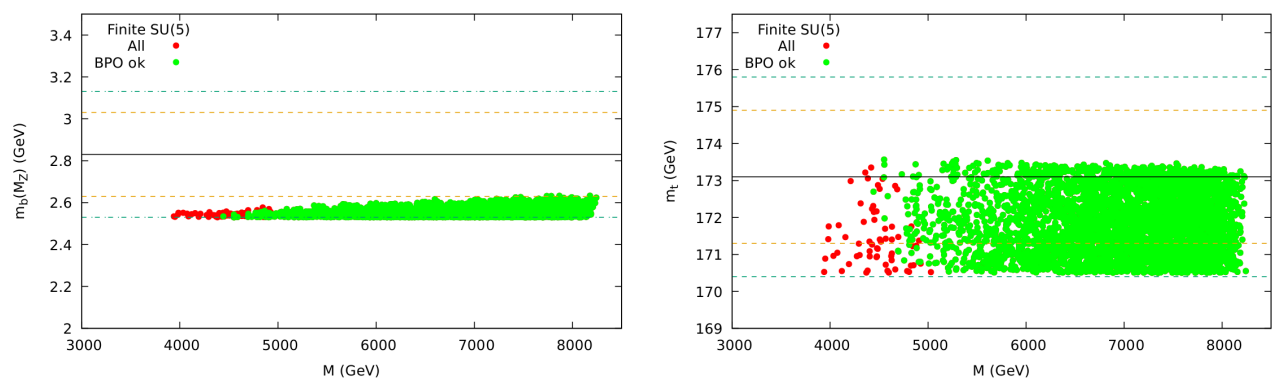

Figure 1: $m_{b}\left(M_{Z}\right)$ (left) and $m_{t}$ (right) as a function of $M$ for the Finite $N=1 S U(5)$. Green points satisfy the $B$-physics constraints.

The light Higgs boson mass is given in Fig. 2 (left), while its theory uncertainty [26] is given in Fig. 2 (right). This point-by-point uncertainty (calculated with FeynHiggs) drops significantly (wrt past analyses) to $0.65-0.70 \mathrm{GeV}$.
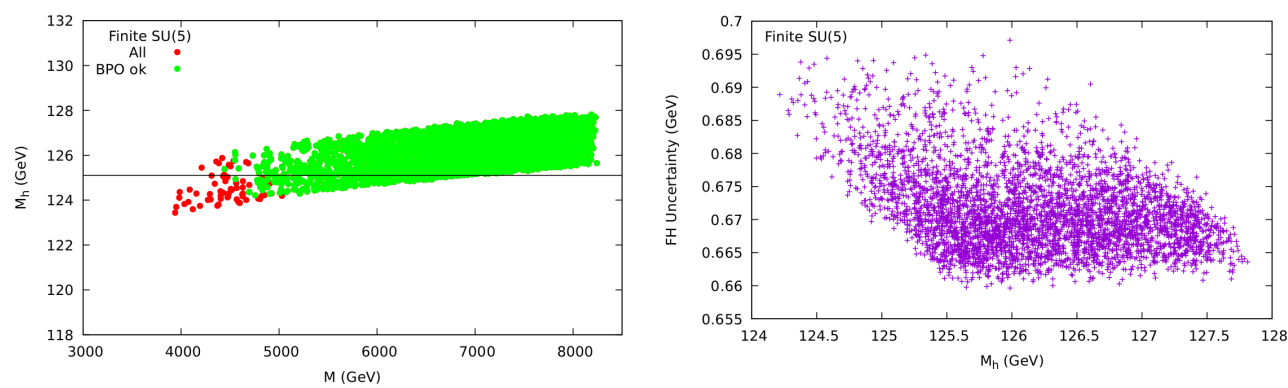

Figure 2: Left: $M_{h}$ as a function of $M$. Green points comply with B-physics constraints. Right: The lightest Higgs mass theoretical uncertainty calculated with FeynHiggs 2.16.0 [26].

\begin{tabular}{|l|rrrrrrrrr|}
\hline & $M_{h}$ & $M_{H}$ & $M_{A}$ & $M_{H^{ \pm}}$ & $m_{\tilde{t}_{1}}$ & $m_{\tilde{t}_{2}}$ & $m_{\tilde{b}_{1}}$ & $m_{\tilde{b}_{2}}$ & $m_{\tilde{g}}$ \\
\hline lightest & 124.4 & 5513 & 5513 & 5510 & 5940 & 6617 & 5888 & 6617 & 8819 \\
heaviest & 125.8 & 28121 & 28121 & 28120 & 10486 & 11699 & 10318 & 11686 & 15509 \\
\hline & $m_{\tilde{\tau}_{1}}$ & $m_{\tilde{\tau}_{2}}$ & $m_{\tilde{\chi}_{1}^{ \pm}}$ & $m_{\tilde{\chi}_{2}^{ \pm}}$ & $m_{\tilde{\chi}_{1}^{0}}$ & $m_{\tilde{\chi}_{2}^{0}}$ & $m_{\tilde{\chi}_{3}^{0}}$ & $m_{\tilde{\chi}_{4}^{0}}$ & $\tan \beta$ \\
\hline lightest & 2225 & 3123 & 3819 & 4801 & 2120 & 3811 & 4820 & 4811 & 50 \\
heaviest & 4215 & 5788 & 7108 & 8200 & 4019 & 7108 & 8227 & 8227 & 51 \\
\hline
\end{tabular}

Table 1: Spectra of the Finite $N=1 S U(5)$. Masses are in $\mathrm{GeV}$ and rounded to $1(0.1) \mathrm{GeV}$ (for the light Higgs mass).

In Tab. 1 we give the lightest and the heaviest spectrum. Compared to our previous analyses [110-117,120-122], the improved evaluation of $M_{h}$ and its uncertainty prefers a heavier (Higgs) spectrum and thus allows only a heavy supersymmetric spectrum. Very heavy coloured SUSY particles are favoured (nearly independent of the $M_{h}$ uncertainty), in agreement with the nonobservation of those particles at the LHC [118]. Overall, the allowed coloured SUSY masses would 
remain unobservable at the (HL-)LHC, the ILC or CLIC. However, the coloured spectrum would be accessible at the FCC-hh [119], as could the full heavy Higgs boson spectrum.

The model has a high relic abundance for CDM. The CDM alternatives proposed for the Minimal $S U(5)$ model in [110] can also be applied here.

\subsection{Two-Loop Finite $N=1 S U(3) \otimes S U(3) \otimes S U(3)$}

We continue our analysis with the two-loop finite $N=1$ supersymmetric $S U(3) \otimes S U(3) \otimes$ $S U$ (3) model, where again below $M_{\mathrm{GUT}}$ we get the MSSM. We take into account two new thresholds for the masses of the new particles at $\sim 10^{13} \mathrm{GeV}$ and $\sim 10^{14} \mathrm{GeV}$ resulting in a wider phenomenologically viable parameter space [123].
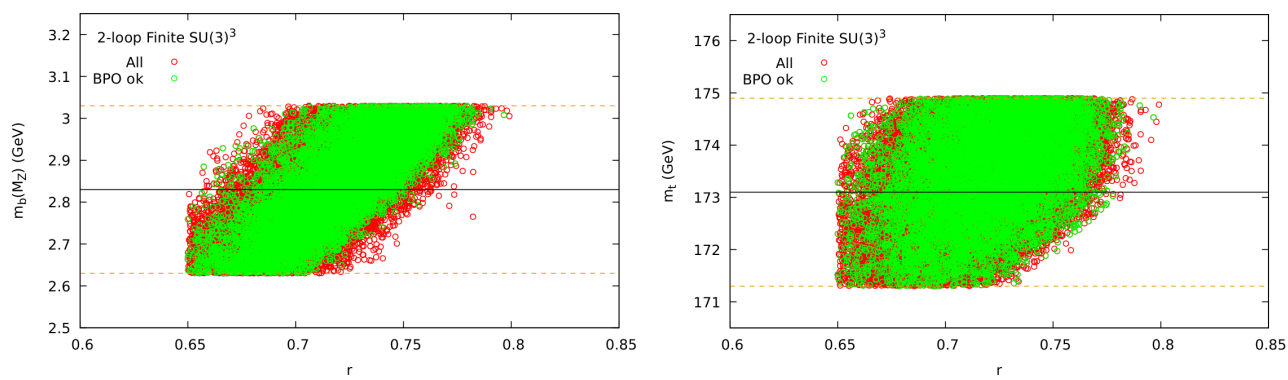

Figure 3: Bottom and top quark masses for the Finite $N=1 S U(3) \otimes S U(3) \otimes S U(3)$ model, with $\mu<0$, as functions of $r$. The black horizontal line is the experimental central value, and the dashed orange ones are the $2 \sigma$ limits. Green points satisfy $B$-physics constraints.

Looking for the values of the parameter $r$ (see Subsection 4.3) which comply with the experimental limits, we find (see Fig. 3) that both masses are in the experimental range for the same value of $r$ between 0.65 and 0.80 (we singled out the $\mu<0$ case as the most promising). The inclusion of the abovementioned thresholds gives an important improvement on the top mass from past versions of the model [83, 124-126].
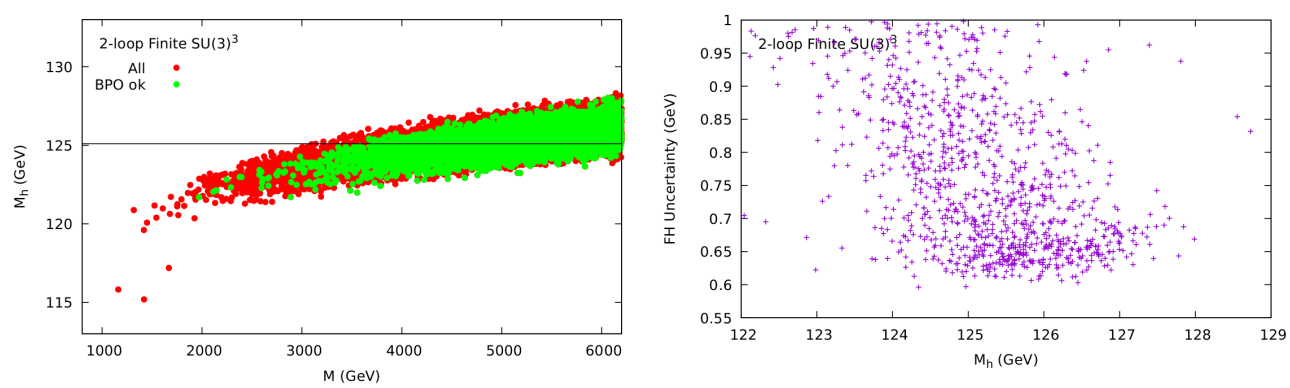

Figure 4: Left: $M_{h}$ as a function of $M$ for the Finite $N=1 S U(3) \otimes S U(3) \otimes S U(3)$. Right: The Higgs mass theoretical uncertainty [26].

In Fig. 4 (left) we show the light Higgs boson mass, while the point-by-point calculated theoretical uncertainty is presented in Fig. 4 (right). Tab. 2 gives the lightest and heaviest spectrum. All constraints regarding quark masses, the light Higgs boson mass and B-physics are satisfied, 


\begin{tabular}{|c|rrrrrrrrr|}
\hline & $M_{h}$ & $M_{H}$ & $M_{A}$ & $M_{H^{ \pm}}$ & $m_{\tilde{t}_{1}}$ & $m_{\tilde{t}_{2}}$ & $m_{\tilde{b}_{1}}$ & $m_{\tilde{b}_{2}}$ & $m_{\tilde{g}}$ \\
\hline lightest & 124.2 & 1918 & 1918 & 1917 & 4703 & 5480 & 4671 & 6013 & 6329 \\
heaviest & 125.9 & 12053 & 12053 & 12050 & 10426 & 10631 & 10426 & 11193 & 14550 \\
\hline & $m_{\tilde{\tau}_{1}}$ & $m_{\tilde{\tau}_{2}}$ & $m_{\tilde{\chi}_{1}^{ \pm}}$ & $m_{\tilde{\chi}_{2}^{ \pm}}$ & $m_{\tilde{\chi}_{1}^{0}}$ & $m_{\tilde{\chi}_{2}^{0}}$ & $m_{\tilde{\chi}_{3}^{0}}$ & $m_{\tilde{\chi}_{4}^{0}}$ & $\tan \beta$ \\
\hline lightest & 1774 & 2694 & 2736 & 5469 & 1517 & 2736 & 5480 & 5481 & 44 \\
heaviest & 5999 & 7113 & 6713 & 10522 & 3767 & 6703 & 10522 & 10523 & 53 \\
\hline
\end{tabular}

Table 2: $\quad$ Spectra of the Finite $N=1 S U(3) \otimes S U(3) \otimes S U(3)$. Masses are in GeV and rounded to 1 (0.1) GeV (for the light Higgs mass).

rendering making the model very successful. The accessibility of the heavier (coloured) spectrum will be subject to future colliders.

\subsection{Reduced MSSM}

For the analysis of our final model we choose the unification scale to apply the corrections to all these RGI relations. The full discussion on the selection of the free parameters of the model can be found in [110]. In total, we vary $\rho_{\tau}, \rho_{h_{\tau}}, M$ and $\mu$.

In Fig. 5 we see the model's predictions for the bottom and top mass respectively. The horizontal lines denote the $2 \sigma$ level uncertainty. Next, $M_{h}$ is shown in Fig. 6 (left),while the theory uncertainty given in Fig. 6 (right) has dropped below $1 \mathrm{GeV}$. The Higgs mass predicted by the model lies within the LHC range.
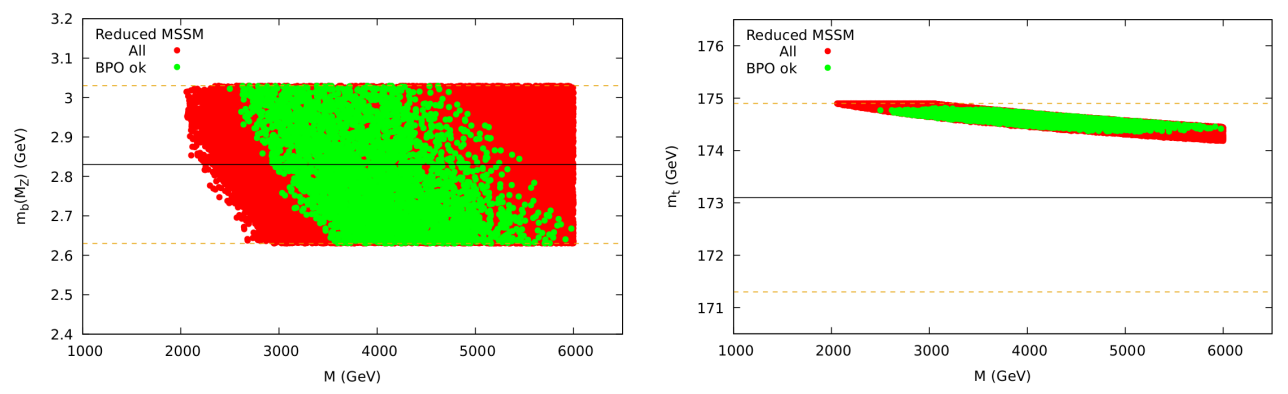

Figure 5: The left (right) plot shows the bottom (top) quark mass for the Reduced MSSM.

The $M_{h}$ limits set a limit on the low-energy supersymmetric masses, rendering the Reduced MSSM highly predictive and testable. In Tab. 3 we show the lightest and heaviest value of each parameter of the supersymmetric spectrum. The HL-LHC [127] will be able to test the full Higgs spectrum. The lighter supersymmetric particles, which are given by the electroweak spectrum, will mostly remain unobservable at the LHC and at future $e^{+} e^{-}$colliders such as the ILC or CLIC. An exception are the lightest neutralino and chargino masses, which could be covered by CLIC3TeV. The coloured mass spectrum will remain unobservable at the (HL-)LHC, but could be accessible at the FCC-hh [119]. 

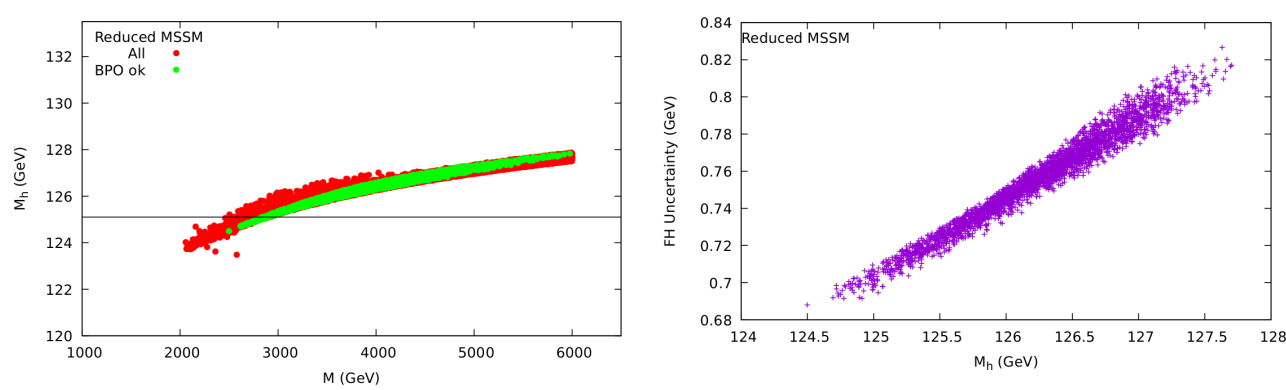

Figure 6: Left: The lightest Higgs boson mass, $M_{h}$ in the Reduced MSSM. The green points is the full model prediction. Right: the lightest Higgs mass theoretical uncertainty [26].

\begin{tabular}{|c|rrrrrrrrr|}
\hline & $M_{h}$ & $M_{H}$ & $M_{A}$ & $M_{H^{ \pm}}$ & $m_{\tilde{t}_{1}}$ & $m_{\tilde{t}_{2}}$ & $m_{\tilde{b}_{1}}$ & $m_{\tilde{b}_{2}}$ & $m_{\tilde{g}}$ \\
\hline lightest & 124.5 & 1305 & 1305 & 1297 & 3851 & 4029 & 3699 & 4007 & 5126 \\
heaviest & 125.8 & 1801 & 1801 & 1780 & 5275 & 5564 & 5076 & 5502 & 7017 \\
\hline & $m_{\tilde{\tau}_{1}}$ & $m_{\tilde{\tau}_{2}}$ & $m_{\tilde{\chi}_{1}^{ \pm}}$ & $m_{\tilde{\chi}_{2}^{ \pm}}$ & $m_{\tilde{\chi}_{1}^{0}}$ & $m_{\tilde{\chi}_{2}^{0}}$ & $m_{\tilde{\chi}_{3}^{0}}$ & $m_{\tilde{\chi}_{4}^{0}}$ & $\tan \beta$ \\
\hline lightest & 1705 & 2536 & 843 & 1875 & 711 & 2579 & 3516 & 3517 & 40 \\
heaviest & 4288 & 6008 & 1004 & 2195 & 1001 & 3666 & 4814 & 4815 & 45 \\
\hline
\end{tabular}

Table 3: Spectra of the Reduced MSSM. All masses are in GeV and rounded to $1(0.1) \mathrm{GeV}$ (for the light Higgs mass).

\section{Conclusions}

We briefly reviewed the ideas concerning the reduction of couplings, then new results were given for three specific models, namely the Finite $N=1 S U(5)$, the Two-Loop Finite $N=1$ $S U(3) \otimes S U(3) \otimes S U(3)$ and the Reduced MSSM. The three models are in natural agreement with all LHC measurements and searches and they predict relatively heavy spectra which evade detection in present and near-future colliders. An exception is the lighter part of the Reduced MSSM spectrum which could be covered by CLIC3TeV. FCC-hh will be able to test the predicted parameter spaces of all models.

\section{Acknowledgements}

GZ thanks the ITP of Heidelberg, MPI Munich, CERN Department of Theoretical Physics, IFT Madrid and MPI-AEI for their hospitality. The work of S.H. is supported in part by the MEINCOP Spain under Contract FPA2016-78022-P, in part by the Spanish Agencia Estatal de Investigación (AEI), the EU Fondo Europeo de Desarrollo Regional (FEDER) through the project FPA201678645-P, in part by the "Spanish Red Consolider MultiDark" FPA2017-90566-REDC, and in part by the AEI through the grant IFT Centro de Excelencia Severo Ochoa SEV-2016-0597. The work of M.M. is partly supported by UNAM PAPIIT through Grant IN111518. The work of G.P., N.T. and G.Z. is partially supported by the COST actions CA15108 and CA16201. GZ has been sup- 
ported within the Excellence Initiative funded by the German and State Governments, at the Institute for Theoretical Physics, Heidelberg University and from the Excellence Grant Enigmass of LAPTh.

\section{References}

[1] J. Kubo, S. Heinemeyer, M. Mondragon, O. Piguet, K. Sibold, W. Zimmermann and G. Zoupanos, PoS (Higgs \& top)001, Ed. Klaus Sibold, https://pos.sissa.it/cgi-bin/reader/conf.cgi?confid=222. A short version is published in arXiv:1411.7155 [hep-ph].

[2] W. Zimmermann, Commun. Math. Phys. 97 (1985) 211.

[3] R. Oehme, W. Zimmermann, Commun. Math. Phys. 97 (1985) 569.

[4] R. Oehme, Prog. Theor. Phys. Suppl. 86 (1986) 215.

[5] E. Ma, Phys. Rev. D 17 (1978) 623; E. Ma, Phys. Rev. D 31 (1985) 1143.

[6] N. P. Chang, Phys. Rev. D 10 (1974) 2706.

[7] S. Nandi and W. C. Ng, Phys. Rev. D 20 (1979) 972.

[8] J. C. Pati and A. Salam, Phys. Rev. Lett. 31 (1973) 661.

[9] H. Georgi and S. L. Glashow, Phys. Rev. Lett. 32 (1974) 438.

[10] H. Georgi, H. R. Quinn and S. Weinberg, Phys. Rev. Lett. 33 (1974) 451.

[11] H. Fritzsch and P. Minkowski, Annals Phys. 93 (1975) 193.

[12] F. Gursey, P. Ramond and P. Sikivie, Phys. Lett. 60B (1976) 177.

[13] Y. Achiman and B. Stech, Phys. Lett. 77B (1978) 389.

[14] J. Kubo, M. Mondragón, G. Zoupanos, Nucl. Phys. B424 (1994) 291.

[15] D. Kapetanakis, M. Mondragón, G. Zoupanos, Z. Phys. C60 (1993) 181.

[16] M. Mondragón, G. Zoupanos, Nucl. Phys. Proc. Suppl. 37C (1995) 98.

[17] Tevatron Electroweak Working Group, CDF and D0 Collaborations, (2011), 1107.5255.

[18] S. Heinemeyer, M. Mondragón and G. Zoupanos, JHEP 0807 (2008) 135 [arXiv:0712.3630 [hep-ph]].

[19] ATLAS Collaboration, G. Aad et al., Phys.Lett. B716 (2012) 1, 1207.7214;

[20] ATLAS Collaboration, Reports ATLAS-CONF-2013-014, ATLAS-COM-CONF-2013-025 (2013).

[21] CMS Collaboration, S. Chatrchyan et al., Phys.Lett. B716, 30 (2012), arXiv:1207.7235.

[22] CMS Collaboration, S. Chatrchyan et al., (2013), arXiv:1303.4571.

[23] G. Degrassi, S. Heinemeyer, W. Hollik, P. Slavich and G. Weiglein, Eur. Phys. J. C28, 133 (2003), [hep-ph/0212020].

[24] H. Bahl, S. Heinemeyer, W. Hollik and G. Weiglein, Eur. Phys. J. C 78 (2018) no.1, 57 [arXiv:1706.00346 [hep-ph]]. 
[25] S. Heinemeyer, W. Hollik and G. Weiglein, Comput. Phys. Commun. 124 (2000) 76 [hep-ph/9812320]; S. Heinemeyer, W. Hollik and G. Weiglein, Eur. Phys. J. C 9 (1999) 343 [hep-ph/9812472]; M. Frank, T. Hahn, S. Heinemeyer, W. Hollik, H. Rzehak and G. Weiglein, JHEP 0702 (2007) 047 [hep-ph/0611326]; T. Hahn, S. Heinemeyer, W. Hollik, H. Rzehak and G. Weiglein, Comput. Phys. Commun. 180 (2009) 1426. T. Hahn, S. Heinemeyer, W. Hollik, H. Rzehak and G. Weiglein, Phys. Rev. Lett. 112 (2014) no.14, 141801 [arXiv:1312.4937 [hep-ph]]; H. Bahl and W. Hollik, Eur. Phys. J. C 76 (2016) no.9, 499 [arXiv:1608.01880 [hep-ph]]; H. Bahl, T. Hahn, S. Heinemeyer, W. Hollik, S. Paßehr, H. Rzehak and G. Weiglein, arXiv:1811.09073 [hep-ph]; See http://www. feynhiggs.de.

[26] H. Bahl, S. Heinemeyer, W. Hollik and G. Weiglein, arXiv:1912.04199 [hep-ph].

[27] J. Kubo, K. Sibold and W. Zimmermann, Nucl. Phys. B259, 331 (1985).

[28] J. Kubo, K. Sibold and W. Zimmermann, Phys. Lett. B220, 185 (1989).

[29] O. Piguet and K. Sibold, Phys. Lett. B 229 (1989) 83.

[30] J. Kubo, M. Mondragón and G. Zoupanos, Phys. Lett. B 389 (1996) 523 [hep-ph/9609218].

[31] P. Breitenlohner and D. Maison, Commun. Math. Phys. 219 (2001) 179.

[32] W. Zimmermann, Commun. Math. Phys. 219 (2001) 221.

[33] A. Parkes and P. C. West, Phys. Lett. 138B (1984) 99.

[34] P. C. West, Phys. Lett. 137B (1984) 371.

[35] D. R. T. Jones and A. J. Parkes, Phys. Lett. 160B (1985) 267.

[36] D. R. T. Jones and L. Mezincescu, Phys. Lett. 138B (1984) 293.

[37] A. J. Parkes, Phys. Lett. 156B (1985) 73.

[38] J. Wess and B. Zumino, Phys. Lett. 49B (1974) 52.

[39] J. Iliopoulos and B. Zumino, Nucl. Phys. B 76 (1974) 310.

[40] K. Fujikawa and W. Lang, Nucl. Phys. B 88 (1975) 61.

[41] R. Delbourgo, Nuovo Cim. A 25 (1975) 646.

[42] A. Salam and J. A. Strathdee, Nucl. Phys. B 86 (1975) 142.

[43] M. T. Grisaru, W. Siegel and M. Rocek, Nucl. Phys. B 159 (1979) 429.

[44] L. Girardello and M. T. Grisaru, Nucl. Phys. B 194 (1982) 65.

[45] Y. Yamada, Phys. Rev. D 50 (1994) 3537 [hep-ph/9401241].

[46] D. I. Kazakov, Phys. Lett. B 421 (1998) 211 [hep-ph/9709465].

[47] I. Jack, D. R. T. Jones and A. Pickering, Phys. Lett. B 426 (1998) 73 [hep-ph/9712542].

[48] J. Hisano and M. A. Shifman, Phys. Rev. D 56 (1997) 5475 [hep-ph/9705417].

[49] I. Jack and D. R. T. Jones, Phys. Lett. B 415 (1997) 383 [hep-ph/9709364].

[50] L. V. Avdeev, D. I. Kazakov and I. N. Kondrashuk, Nucl. Phys. B 510 (1998) 289 [hep-ph/9709397].

[51] D. I. Kazakov, Phys. Lett. B 449 (1999) 201 [hep-ph/9812513].

[52] I. Jack and D. R. T. Jones, Phys. Lett. B 465 (1999) 148 [hep-ph/9907255]. 
[53] T. Kobayashi et al., AIP Conf. Proc. 490 (1999) 279.

[54] T. Kobayashi, J. Kubo, G. Zoupanos, Phys. Lett. B427 (1998) 291.

[55] S. Rajpoot and J. G. Taylor, Phys. Lett. B147, 91 (1984).

[56] S. Rajpoot and J. G. Taylor, Int. J. Theor. Phys. 25, 117 (1986).

[57] D. R. T. Jones, L. Mezincescu, Y. P. Yao, Phys. Lett. B148 (1984) 317.

[58] I. Jack, D. R. T. Jones, Phys. Lett. B333 (1994) 372.

[59] L. O’Raifeartaigh, Nucl. Phys. B96, 331 (1975).

[60] P. Fayet and J. Iliopoulos, Phys. Lett. B51, 461 (1974).

[61] C. Lucchesi, O. Piguet, K. Sibold, Phys. Lett. B201 (1988) 241.

[62] C. Lucchesi, O. Piguet, K. Sibold, Helv. Phys. Acta 61 (1988) 321.

[63] O. Piguet and K. Sibold, Int. J. Mod. Phys. A1, 913 (1986).

[64] O. Piguet and K. Sibold, Phys. Lett. B177, 373 (1986).

[65] P. Ensign and K. T. Mahanthappa, Phys. Rev. D36, 3148 (1987).

[66] C. Lucchesi, G. Zoupanos, Fortschr. Phys. 45 (1997) 129.

[67] O. Piguet, hep-th/9606045, talk given at "10th International Conference on Problems of Quantum Field Theory".

[68] S. Dimopoulos and H. Georgi, Nucl. Phys. B193 (1981) 150.

[69] N. Sakai, Zeit. f. Phys. C11 (1981) 153.

[70] J. Kubo, M. Mondragón and G. Zoupanos, Nucl. Phys. B424 (1994) 291.

[71] N. Polonsky and A. Pomarol, Phys. Rev. Lett. 73 (1994) 2292.

[72] D. I. Kazakov, M. Y. Kalmykov, I. N. Kondrashuk and A. V. Gladyshev, Nucl. Phys. B 471 (1996) 389

[73] J. Kubo, M. Mondragon, M. Olechowski and G. Zoupanos, Nucl. Phys. B 479 (1996), 25-45 [arXiv:hep-ph/9512435 [hep-ph]].

[74] J. Kubo, M. Mondragón, N. D. Tracas, G. Zoupanos, Phys. Lett. B342 (1995) 155.

[75] J. Kubo, M. Mondragon, M. Olechowski and G. Zoupanos, "Gauge Yukawa unification and the top bottom hierarchy", hep-ph/9510279.

[76] J. Kubo, M. Mondragón, G. Zoupanos, Acta Phys. Polon. B27 (1997) 3911-3944.

[77] T. Kobayashi, J. Kubo, M. Mondragón, G. Zoupanos, Nucl. Phys. B511 (1998) 45.

[78] M. Mondragon and G. Zoupanos, J. Phys. Conf. Ser. 171 (2009) 012095.

[79] J. Leon, J. Perez-Mercader, M. Quiros and J. Ramirez-Mittelbrunn, Phys. Lett. B156, 66 (1985).

[80] S. Hamidi and J. H. Schwarz, Phys. Lett. B147, 301 (1984).

[81] D. R. T. Jones and S. Raby, Phys. Lett. B143, 137 (1984).

[82] K. S. Babu, T. Enkhbat and I. Gogoladze, Phys. Lett. B 555, 238 (2003) [hep-ph/0204246].

[83] E. Ma, M. Mondragón, and G. Zoupanos, JHEP 12, 026 (2004), hep-ph/0407236. 
[84] A. De Rújula, H. Georgi, and S. L. Glashow, p. 88 (1984), Fifth Workshop on Grand Unification, K. Kang, H. Fried, and P. Frampton eds., World Scientific, Singapore.

[85] G. Lazarides, C. Panagiotakopoulos, and Q. Shafi, Phys. Lett. B315, 325 (1993), hep-ph/9306332.

[86] G. Lazarides and C. Panagiotakopoulos, Phys. Lett. B336, 190 (1994), hep-ph/9403317.

[87] E. Ma, Phys. Rev. D36, 274 (1987).

[88] N. Irges and G. Zoupanos, Phys. Lett. B 698 (2011) 146

[89] N. Irges, G. Orfanidis and G. Zoupanos, PoS CORFU 2011 (2011) 105

[90] M. Mondragón, N. D. Tracas and G. Zoupanos, Phys. Lett. B 728 (2014) 51 [arXiv:1309.0996 [hep-ph]].

[91] M. Mondragón, S. Heinemeyer, N. Tracas and G. Zoupanos, PoS CORFU2016 (2017) 041.

[92] M. Mondragón, N.D. Tracas, G. Zoupanos, Phys. Lett. B 728, 51 (2014).

[93] S. Heinemeyer, M. Mondragón, N. Tracas and G. Zoupanos, JHEP 1808 (2018) 150.

[94] M. Tanabashi et al. [Particle Data Group], Phys. Rev. D 98 (2018) no.3, 030001.

[95] S. Chatrchyan et al. [CMS Collaboration], Phys. Lett. B 716 (2012) 30 [arXiv:1207.7235 [hep-ex]].

[96] S. Heinemeyer, O. Stal and G. Weiglein, Phys. Lett. B 710 (2012) 201 [arXiv:1112.3026 [hep-ph]].

[97] P. Bechtle, S. Heinemeyer, O. Stal, T. Stefaniak, G. Weiglein and L. Zeune, Eur. Phys. J. C 73 (2013) no.4, 2354 [arXiv:1211.1955 [hep-ph]].

[98] P. Bechtle, H. E. Haber, S. Heinemeyer, O. Stal, T. Stefaniak, G. Weiglein and L. Zeune, Eur. Phys. J. C 77 (2017) no.2, 67 [arXiv:1608.00638 [hep-ph]].

[99] M. Misiak et al., Phys. Rev. Lett. 98 (2007) 022002 [hep-ph/0609232];

M. Ciuchini, G. Degrassi, P. Gambino and G. F. Giudice, Nucl. Phys. B 534 (1998) 3 [hep-ph/9806308];

G. Degrassi, P. Gambino and G. F. Giudice, JHEP 0012 (2000) 009 [hep-ph/0009337];

M. Carena, D. Garcia, U. Nierste and C. E. M. Wagner, Phys. Lett. B 499 (2001) 141 [hep-ph/0010003];

G. D’Ambrosio, G. F. Giudice, G. Isidori and A. Strumia, Nucl. Phys. B 645 (2002) 155 [hep-ph/0207036].

[100] D. Asner et al. [Heavy Flavor Averaging Group], arXiv:1010.1589 [hep-ex].

[101] C. Bobeth, M. Gorbahn, T. Hermann, M. Misiak, E. Stamou and M. Steinhauser, Phys. Rev. Lett. 112 (2014) 101801 [arXiv:1311.0903 [hep-ph]];

T. Hermann, M. Misiak and M. Steinhauser, JHEP 1312 (2013) 097 [arXiv:1311.1347 [hep-ph]];

C. Bobeth, M. Gorbahn and E. Stamou, Phys. Rev. D 89 (2014) no.3, 034023 [arXiv:1311.1348 [hep-ph]].

[102] A. J. Buras, Phys. Lett. B 566 (2003) 115 [hep-ph/0303060]; G. Isidori and D. M. Straub, Eur. Phys. J. C 72 (2012) 2103 [arXiv:1202.0464 [hep-ph]].

[103] R. Aaij et al. [LHCb Collaboration], Phys. Rev. Lett. 110 (2013) 021801 [arXiv:1211.2674 [Unknown]].

[104] S. Chatrchyan et al. [CMS Collaboration], Phys. Rev. Lett. 111 (2013) 101804 [arXiv:1307.5025 [hep-ex]]. 
[105] CMS and LHCb Collaborations [CMS and LHCb Collaborations], CMS-PAS-BPH-13-007, LHCb-CONF-2013-012, CERN-LHCb-CONF-2013-012.

[106] G. Isidori and P. Paradisi, Phys. Lett. B 639 (2006) 499 [hep-ph/0605012]; G. Isidori, F. Mescia, P. Paradisi and D. Temes, Phys. Rev. D 75 (2007) 115019 [hep-ph/0703035 [HEP-PH]].

[107] K. A. Olive et al. [Particle Data Group], Chin. Phys. C 38 (2014) 090001.

[108] A. J. Buras, P. Gambino, M. Gorbahn, S. Jager and L. Silvestrini, Nucl. Phys. B 592 (2001) 55 [hep-ph/0007313].

[109] R. Aaij et al. [LHCb Collaboration], New J. Phys. 15 (2013) 053021 [arXiv:1304.4741 [hep-ex]].

[110] S. Heinemeyer, M. Mondragón, G. Patellis, N. Tracas and G. Zoupanos, [arXiv:2002.10983 [hep-ph]].

[111] S. Heinemeyer, M. Mondragón and G. Zoupanos, Phys. Lett. B 718 (2013) 1430 [arXiv:1211.3765 [hep-ph]].

[112] S. Heinemeyer, M. Mondragón and G. Zoupanos, Int. J. Mod. Phys. Conf. Ser. 13 (2012) 118.

[113] S. Heinemeyer, M. Mondragón and G. Zoupanos, Phys. Part. Nucl. 44 (2013) 299.

[114] S. Heinemeyer, M. Mondragón, G. Patellis, N. Tracas and G. Zoupanos, Symmetry 10 (2018) no.3, 62 [arXiv:1802.04666 [hep-ph]].

[115] S. Heinemeyer, M. Mondragon, G. Patellis, N. Tracas and G. Zoupanos, PoS CORFU 2017 (2018) 081.

[116] S. Heinemeyer, M. Mondragón, N. Tracas and G. Zoupanos, Phys. Rept. 814 (2019) 1 [arXiv:1904.00410 [hep-ph]].

[117] S. Heinemeyer, M. Mondragon, G. Patellis, N. Tracas and G. Zoupanos, PoS CORFU 2018 (2019) 077.

[118] https://twiki.cern.ch/twiki/bin/view/AtlasPublic/SupersymmetryPublicResults, https://twiki.cern.ch/twiki/bin/view/CMSPublic/PhysicsResultsSUS

[119] M. Mangano, CERN Yellow Report CERN 2017-003-M [arXiv:1710.06353 [hep-ph]].

[120] S. Heinemeyer, M. Mondragón and G. Zoupanos, SIGMA 6 (2010) 049 [arXiv:1001.0428 [hep-ph]].

[121] S. Heinemeyer, M. Mondragón and G. Zoupanos, Fortsch. Phys. 61 (2013) no.11, 969 [arXiv:1305.5073 [hep-ph]].

[122] S. Heinemeyer, M. Mondragón, N. Tracas and G. Zoupanos, Nucl. Phys. B 927 (2018) 319.

[123] M. Mondragon and G. Zoupanos, Phys. Part. Nucl. Lett. 8 (2011) 173.

[124] S. Heinemeyer, E. Ma, M. Mondragon and G. Zoupanos, AIP Conf. Proc. 1200 (2010) no.1, 568 [arXiv:0910.0501 [hep-ph]].

[125] S. Heinemeyer, E. Ma, M. Mondragon and G. Zoupanos, J. Phys. Conf. Ser. 259 (2010) 012097.

[126] S. Heinemeyer, E. Ma, M. Mondragon and G. Zoupanos, Fortsch. Phys. 58 (2010) 729.

[127] CMS Collaboration, CMS-DP-2016-064. 